in vivo $34: 2587-2593(2020)$

doi:10.21873/invivo.12076

\title{
Intensity-modulated Radiation Therapy for Lymph Node Oligo-recurrence
}

\author{
AKIKO SATO $^{1,2}$, MOTOKO OMURA ${ }^{1}$, YUMIKO MINAGAWA $^{1}$, KENGO MATSUI $^{1}$, \\ RYOSUKE SHIRATA ${ }^{1}$, HIDEYUKI HONGO ${ }^{1}$, HARUMITSU HASHIMOTO ${ }^{3}$, \\ TOSHIHIRO MISUMI ${ }^{4}$, TOMIO INOUE ${ }^{5}$ and MASAHARU HATA ${ }^{2}$ \\ ${ }^{I}$ Department of Radiation Oncology, Shonan Kamakura General Hospital, Kamakura, Japan; \\ ${ }^{2}$ Department of Radiation Oncology, Yokohama City University Graduate School of Medicine, Yokohama, Japan; \\ ${ }^{3}$ Department of Radiation Oncology, Shonan Fujisawa Tokushukai Hospital, Fujisawa, Japan; \\ ${ }^{4}$ Department of Biostatistics, Yokohama City University School of Medicine, Yokohama, Japan; \\ ${ }^{5}$ Department of Advanced Medical Center, Shonan Kamakura General Hospital, Kamakura, Japan
}

\begin{abstract}
Background/Aim: This study aimed to evaluate the effect of intensity-modulated radiation therapy (IMRT) on the clinical outcomes of patients with lymph node (LN) oligo-recurrence and a controlled primary tumor. Patients and Methods: We retrospectively reviewed the medical records of 21 patients diagnosed with LN oligo-recurrence who received IMRT with curative intent. Patients with tumor of various primary sites and histopathological types were included in this study. Results: The 3-year overall survival $(O S)$ and in-field progression-free survival (PFS) rates were $75 \%$ and $52 \%$, respectively. Statistical analysis showed that lower dose to the gross tumor volume (GTV) and larger GTV were significantly associated with poorer OS; adenocarcinoma and lower dose to GTV were significantly associated with poorer in-field PFS. No patients experienced severe adverse events. Conclusion: IMRT may provide a safe and effective treatment for patients with LN oligo-recurrence. Tumor dose-escalation sparing normal tissue using IMRT technology may improve the OS and in-field PFS.
\end{abstract}

Radical local therapy is generally not considered for patients with cancer metastasis or recurrence because the cancer has already spread throughout the body at the time of diagnosis and is thus difficult to cure. However, an increasing number

This article is freely accessible online.

Correspondence to: Akiko Sato, Department of Radiation Oncology, Yokohama City University Graduate School of Medicine, 3-9 Fukuura, Kanazawa-ku, Yokohama, 236-0004, Japan. Tel: +81 457872800, Fax: +81 457860369 e-mail: akikosato.tk@ gmail.com

Key Words: Cancer, intensity-modulated radiation therapy, lymph nodes, oligometastases, oligo-recurrence, radiation therapy. of reports have suggested that the prognosis of these patients can be improved by local therapies such as surgery or radiotherapy if the number of affected regions is limited. This pathological condition was first defined as oligometastases by Hellman and Weichselbaum in 1995 (1). They suggested that for many cancer types, a few metastases initially exist during the oligometastatic phase, after which the malignant cells acquire widespread metastatic potential (2). Theoretically, delivery of radical local therapy during the oligometastatic phase might improve the prognosis of patients who would otherwise be offered exclusively systemic therapy or palliative care.

Oligo-recurrence, as a specific oligometastatic situation, was a concept proposed by Niibe in 2006. In oligorecurrence, the primary site should be controlled and all gross metastatic lesions can be treated by local therapy with radical intent (3). In contrast, oligometastases is defined as a few metastases regardless of the status of the primary lesion. Patients with oligo-recurrence are more likely to be cured compared with those with oligometastases.

High-precision radiation therapies, such as stereotactic radiotherapy (SRT) and intensity-modulated radiation therapy (IMRT), have undergone rapid developments in recent years. SRT is a multi-directional radiation therapy for localized tumors that achieves effective tumor control by providing a high dose in a small fraction focused on a small lesion. SRT has demonstrated good local control for oligometastatic lesions of around $80 \%$ (2). However, it is limited to small lesions (diameter $<3 \mathrm{~cm}$ ), considering the balance between the antitumor effects and adverse effects on the surrounding normal tissues. IMRT was developed from conventional three-dimensional conformal RT technology. IMRT can provide an adequate dose to the target whilst sparing the normal organs at risk (OARs), even if the target is large or has a complex shape, such as the lymph node 
(LN) area. IMRT may thus be more widely applicable than SRT. However, reports on the use of IMRT for oligorecurrence are still lacking.

In this study, we retrospectively evaluated the treatment outcomes and prognostic factors in patients with LN oligorecurrence treated with IMRT.

\section{Patients and Methods}

Patients and clinical practice. We retrospectively reviewed the medical records of 21 patients diagnosed with $\mathrm{LN}$ oligo-recurrence who received IMRT between October 2010 and August 2017 at Shonan Kamakura General Hospital. The inclusion criteria were as follows: oligo-recurrence occurred exclusively in LNs, all detectable involved LNs were treated with curative intent, and the primary lesion was controlled.

All primary tumors were confirmed histopathologically. The recurrent LN lesion was confirmed by biopsy in one patient, and was diagnosed by imaging methods and based on the clinical course in the other patients. All patients underwent a workup including basic laboratory studies, contrast-enhanced computed tomographic (CT) scans of the neck to the pelvis, and magnetic resonance imaging of the lesions in some cases. In addition, some patients underwent whole-body positron-emission CT.

This study was approved by the Review Board of our Institution (CRB3180005), and informed consent was obtained from all patients.

Treatment planning. For RT planning, axial images with a slice thickness of $2 \mathrm{~mm}$ were acquired using a 64-row multi-detector CT system (SOMATOM Sensation 64/Cardiac; Siemens AG, Forchheim, Germany). Contours for targets and OARs were drawn using the Pinnacle system (Version 9.2; Philips Medical Systems, Fitchburg, WI, USA). The gross tumor volume (GTV) included all detectable involved lymph nodes, and the clinical target volume (CTV) included the LN area thought to be at risk, as necessary. The volumes were determined by CT, positron-emission tomography$\mathrm{CT}$, or magnetic resonance imaging. The planning target volume (PTV) was delineated by margins of $5 \mathrm{~mm}$ around the GTV and CTV. When the PTV included the LN area, a boost plan focused on the GTV was applied after the initial plan.

Treatment plans were generated using Hi-Art system TomoTherapy (Accuray Inc., Sunnyvale, CA, USA) inverse planning software based on superposition dose calculation. A field width of $2.5 \mathrm{~cm}$ was applied, and the modulation factor was mainly in the range of 1.5-2.0.

The planning goals were as follows: i) The prescribed dose was defined for the $50 \%$ isodose of the PTV; ii) $95 \%$ of the PTV to receive $\geq 95 \%$ of the prescribed dose; and iii) $80-90 \%$ of the GTV to receive $\geq 100 \%$ of the prescribed dose. The dose to the OAR around the PTV was gradually restricted to the lowest possible dose. For image-guided RT, a megavolt CT scan was performed daily immediately before irradiation.

Evaluation criteria and statistical analysis. Regular follow-up visits were carried out to assess treatment outcome and tolerance. Our institutional standards for patient assessment included physical examination, results of blood tests including tumor markers, and imaging studies every 3-6 months. Progression-free survival (PFS) was calculated as the time from the start of IMRT for oligo-
Table I. Patient, tumor and treatment characteristics of the 21 patients with LN oligo-recurrence.

\begin{tabular}{|c|c|c|}
\hline Characteristic & Characteristic & $\mathrm{n}(\%)$ \\
\hline $\begin{array}{l}\text { Interval between primary } \\
\text { treatment and diagnosis of } \\
\text { oligo-recurrence, months }\end{array}$ & Median (range) & $18.4(6.8-177)$ \\
\hline Age at IMRT for & Median (range) & $67(39-81)$ \\
\hline oligo-recurrence, years & $\geq 70$ Years & $7(33 \%)$ \\
\hline \multirow[t]{2}{*}{ Gender, n (\%) } & Female & $11(52 \%)$ \\
\hline & Male & $10(48 \%)$ \\
\hline \multirow[t]{5}{*}{ Primary site, n (\%) } & Lung & $5(24 \%)$ \\
\hline & Breast & $4(19 \%)$ \\
\hline & Uterus [cervix/corpus] 4 & $4[3 / 1](19 \%)$ \\
\hline & Esophagus & $2(9.5 \%)$ \\
\hline & Other & $6(29 \%)$ \\
\hline \multirow[t]{5}{*}{ Histopathology, n (\%) } & Histopathology & \\
\hline & Adenocarcinoma & $8(38 \%)$ \\
\hline & SCC & $5(24 \%)$ \\
\hline & Invasive ductal carcinoma & $3(14 \%)$ \\
\hline & Other & $5(24 \%)$ \\
\hline \multirow[t]{5}{*}{$\begin{array}{l}\text { Initial treatment for } \\
\text { primary lesion, n }(\%)\end{array}$} & $\begin{array}{l}\text { Surgery with systemic } \\
\text { therapy }\end{array}$ & $10(48 \%)$ \\
\hline & Surgery alone & $7(33 \%)$ \\
\hline & RT alone & $2(10 \%)$ \\
\hline & Surgery and CCRT & $1(5 \%)$ \\
\hline & CCRT & $1(5 \%)$ \\
\hline \multirow{3}{*}{$\begin{array}{l}\text { Site of recurrent } \mathrm{LN} \\
\text { lesions, } \mathrm{n}(\%)\end{array}$} & Regional LN & $14(67 \%)$ \\
\hline & Distant metastasis & $6(29 \%)$ \\
\hline & Both & $1(5 \%)$ \\
\hline \multirow[t]{5}{*}{ Irradiation field, $\mathrm{n}$} & Hilum/mediastinum & 7 \\
\hline & Abdomen & 5 \\
\hline & Pelvis & 3 \\
\hline & Supraclavicular & 3 \\
\hline & Other & 3 \\
\hline \multirow[t]{2}{*}{ RT fields per patient, $\mathrm{n}(\%)$} & Single & $20(95 \%)$ \\
\hline & Two & $1(5 \%)$ \\
\hline \multirow[t]{2}{*}{ Involved LNs per field } & Single & $13(62 \%)$ \\
\hline & Multiple (2-5) & $8(38 \%)$ \\
\hline \multirow[t]{2}{*}{ Involved LNs per field, $\mathrm{n}$} & Median (range) & $9.6(1.4-80)$ \\
\hline & $\geq 20$ & $7(33 \%)$ \\
\hline \multirow{2}{*}{$\begin{array}{l}\text { Regional LN area within RT } \\
\text { field }\end{array}$} & Yes & $17(81 \%)$ \\
\hline & No & $4(19 \%)$ \\
\hline PTV regional LN area, $\mathrm{cm} 3$ & Median (range) & 404 (112-934) \\
\hline Dose to GTV, Gy & Median (range) & $60(50-70)$ \\
\hline Dose to regional LN area, Gy & Median (range) & $44(38-50)$ \\
\hline Systemic therapy for & Yes & $10(48 \%)$ \\
\hline oligo-recurrence, $\mathrm{n}(\%)^{*}$ & No & $11(52 \%)$ \\
\hline
\end{tabular}

IMRT: Intensity-modulated radiation therapy; SCC: squamous cell carcinoma; CCRT: concurrent chemoradiotherapy; LN: lymph node; GTV: gross tumor volume; RT: radiotherapy. *Ten patients received systemic therapy during the period, considered to affect the therapeutic effect of IMRT; six received chemotherapy and four received hormone therapy.

recurrence to the diagnosis of progression or the last follow-up visit if no progression had occurred. Evaluation of treatment response was carried out according to the Response Evaluation Criteria in Solid Tumors (RECIST) (4). 
Toxicities associated with radiation therapy were evaluated using the Common Terminology Criteria for Adverse Events v4.0 (5). Acute toxicity was defined as therapy-related adverse events occurring within 3 months after the beginning of treatment, and late toxicity as those occurring after 3 months.

The overall survival (OS), in-field PFS, and out-of-field PFS rates were estimated by the Kaplan-Meier method. The Cox hazard regression model was used for univariate and multivariate analyses. In the multivariate analysis, a backward elimination procedure was used to identify prognostic factors. Differences between subgroups were analyzed by log-rank tests. For all analyses, values of $p<0.05$ were considered statistically significant. All analyses were performed using JMP ${ }^{\circledR}$ pro12.2.0. (SAS Institute, Cary, NC, USA).

\section{Results}

Patient characteristics and treatment. The patient characteristics and treatment details are given in Table I. The median age at the start of IMRT for oligo-recurrence lesions was 67 years (range $=39-81$ years). The median GTV was 9.6 $\mathrm{cm}^{3}$ (range $=1.4-80.0 \mathrm{~cm}^{3}$ ). Eight patients had a GTV $>14$ $\mathrm{cm}^{3}$ (equivalent to a sphere $3 \mathrm{~cm}$ in diameter), including two patients with tumors located close to the intestine. Four patients $(19.0 \%)$ received IMRT focused on only the GTV, whereas the remaining 17 patients $(81.0 \%)$ received IMRT including the regional $\mathrm{LN}$ area. The median IMRT dose to the GTV was 60 Gy (range=50-70 Gy). All patients completed the course of IMRT. The median follow-up period after IMRT was 33.7 months (range=6.2-71.3 months).

Progression patterns and survival outcomes. At the last follow-up, nine out of the 21 patients (43\%) had achieved disease-free status and the remaining 12 patients (57\%) had developed disease progression. During the follow-up period, two patients had exclusively in-field progression, three had out-of-field progression, and seven had both.

Among the nine patients with in-field progression, only one had a newly arising tumor within the irradiated LN area without regrowth of the original GTV, and the remaining eight had progression exclusively of the GTV. The survival curves are shown in Figure 1. The 3-year OS, in-field PFS, and out-of-field PFS rates were $75 \%, 52 \%$, and $44 \%$, respectively.

Radiation therapy of all four patients with adenocarcinoma treated with a dose to GTV of $\leq 60$ Gy failed in terms of infield control, compared with two out of the eight patients with non-adenocarcinoma treated with the same doses.

Statistical analyses. We analyzed the effects of the following potential prognostic factors on progression and survival: Age, sex, histopathological type, GTV, interval between initial treatment for primary lesion and diagnosis of oligorecurrence, site of $\mathrm{LN}$ regions (regional or distant metastases), irradiation field (GTV only or including LN
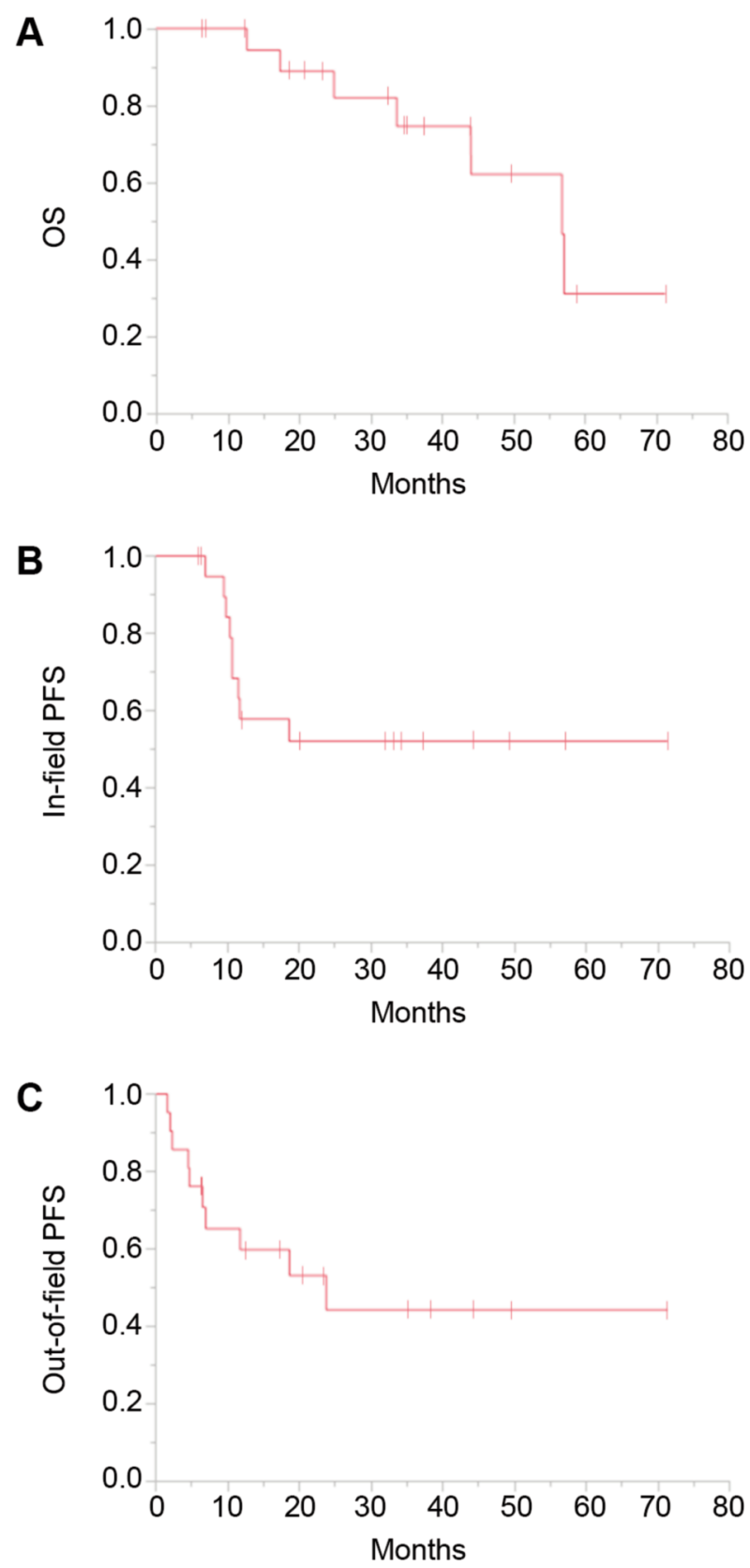

Figure 1. Kaplan-Meier plots of overall survival $(O S)(A)$, in-field progression-free survival (PFS) (B), and out-of-field PFS (C) for all 21 patients.

area), application of systemic therapy, and IMRT dose. The results of univariate and multivariate Cox regression analyses for OS, in-field PFS, and out-of-field PFS are shown in Table II. Univariate Cox regression analysis of OS identified a lower dose to GTV and larger GTV as being significantly associated with poorer OS. Multivariate Cox regression 
Table II. Cox regression analysis of risk factors affecting overall survival (OS), in-field progression-free survival (PFS), and out-of-field PFS.

\begin{tabular}{|c|c|c|c|}
\hline \multirow[t]{2}{*}{ Parameter } & \multicolumn{3}{|c|}{ Univariate analysis } \\
\hline & HR & $95 \% \mathrm{CI}$ & $p$-Value \\
\hline \multicolumn{4}{|l|}{ OS } \\
\hline $\begin{array}{l}\text { Interval between primary } \\
\text { treatment and diagnosis of } \\
\text { oligo-recurrence }\end{array}$ & 0.980 & $0.928-1.003$ & 0.114 \\
\hline Age & 0.963 & $0.885-1.063$ & 0.428 \\
\hline Gender (male) & 0.345 & $0.048-1.744$ & 0.208 \\
\hline Adenocarcinoma & 1.838 & $0.401-9.419$ & 0.426 \\
\hline $\mathrm{SCC}$ & NA & NA & NA \\
\hline Site of LN lesions (regional only) & 0.420 & $0.022-2.483$ & 0.377 \\
\hline GTV & 1.039 & $1.007-1.082$ & 0.017 \\
\hline Including LN area & 0.814 & $0.120-15.92$ & 0.857 \\
\hline Dose to GTV & 0.812 & $0.652-0.972$ & 0.022 \\
\hline Systemic therapy & 0.850 & $0.163-3.948$ & 0.833 \\
\hline \multicolumn{4}{|l|}{ In-field PFS } \\
\hline $\begin{array}{l}\text { Interval between primary } \\
\text { treatment and diagnosis of } \\
\text { oligo-recurrence }\end{array}$ & 0.980 & $0.932-1.012$ & 0.313 \\
\hline Age & 0.969 & $0.896-1.056$ & 0.460 \\
\hline Gender (male) & 0.824 & $0.213-2.771$ & 0.759 \\
\hline Adenocarcinoma* & 3.761 & $0.978-18.06$ & 0.054 \\
\hline $\mathrm{SCC}$ & 0.267 & $0.014-1.464$ & 0.144 \\
\hline Site of LN lesions (regional only) & 2.742 & $0.741-10.15$ & 0.127 \\
\hline GTV & 1.016 & $0.990-1.040$ & 0.217 \\
\hline Including $\mathrm{LN}$ area & 0.589 & $0.134-4.041$ & 0.538 \\
\hline Dose to GTV** & 1.017 & $0.878-1.182$ & 0.825 \\
\hline Systemic therapy & 1.803 & $0.537-6.969$ & 0.343 \\
\hline \multicolumn{4}{|l|}{ Out-of-field PFS } \\
\hline $\begin{array}{l}\text { Interval between primary } \\
\text { treatment and diagnosis } \\
\text { of oligo-recurrence }\end{array}$ & 1.003 & $0.986-1.015$ & 0.671 \\
\hline Age & 0.926 & $0.867-0.992$ & 0.029 \\
\hline Gender (male) & 0.744 & $0.189-2.630$ & 0.646 \\
\hline Adenocarcinoma & 2.859 & $0.796-11.48$ & 0.107 \\
\hline $\mathrm{SCC}$ & NA & NA & NA \\
\hline Site of LN lesions (regional only) & 0.998 & $0.214-3.623$ & 0.998 \\
\hline GTV & 1.002 & $0.972-1.026$ & 0.865 \\
\hline Including $\mathrm{LN}$ area & 0.897 & $0.223-5.968$ & 0.893 \\
\hline Dose to GTV & 0.989 & $0.849-1.147$ & 0.889 \\
\hline Systemic therapy & 1.486 & $0.409-5.392$ & 0.536 \\
\hline
\end{tabular}

CI: Confidence interval; GTV: gross tumor volume; HR: hazard ratio; LN: lymph node; NA: not applicable; SCC: squamous cell carcinoma. Bold values indicate statistical significance. Estimation of multivariate Cox regression models based on backward elimination for OS and outof-field PFS were not converged due to insufficient sample size, consequently no results were obtained. Multivariate analysis gave significant results with $* \mathrm{HR}=6.2(95 \% \mathrm{CI}=1.452-34.25), p=0.014$; and $* * \mathrm{HR}=0.842(95 \% \mathrm{CI}=0.707-0.985) p=0.032$.

analysis showed that adenocarcinoma and lower dose to GTV were significantly associated with poorer in-field PFS. Univariate Cox regression analysis revealed that younger age was significantly associated with poorer out-of-field PFS.
Estimation of multivariate Cox regression models based on backward elimination for OS and out-of-field PFS were not converged due to insufficient sample size, consequently no results were obtained.

The effects of the potential prognostic factors on survival were analyzed by univariate analysis using the log-rank test (Figure 2). OS was significantly lower in patients with a GTV $>20 \mathrm{~cm}^{3}$ compared with patients with smaller tumors (Figure 2A). In-field PFS was significantly lower in patients with adenocarcinoma compared those with nonadenocarcinoma (Figure 2B). Squamous cell carcinoma was associated with significantly better out-of-field PFS than non-squamous cell carcinoma (Figure 2C), and age $>70$ years was also associated with better out-of-field PFS than age $\leq 70$ years but the difference was not statistically significant $(p=0.05)$ (Figure 2D).

Toxicity. No patients experienced acute or late adverse events grade 3 or more, except for one patient who developed acute grade 3 leukopenia. One patient experienced a grade 2 acute skin reaction and another developed grade 2 esophagitis during treatment. No patients experienced late toxicity of IMRT.

\section{Discussion}

We report the clinical outcomes of 21 patients with LN oligo-recurrence treated with conventional fractionated IMRT. The 3-year OS, in-field PFS, and out-of-field PFS rates were $75 \%, 52 \%$, and $44 \%$, respectively. Lower dose to GTV and larger GTV were significantly associated with poorer OS, while adenocarcinoma and lower dose to GTV were significantly associated with poorer in-field PFS, and older age and squamous cell carcinoma were associated with better out-of-field PFS. Based on these findings, we conclude that IMRT may be a safe and effective strategy in patients with LN oligo-recurrence. To the best of our knowledge, this study provides the first report of the clinical outcomes and prognostic factors in patients treated with IMRT for LN oligo-recurrence from various primary tumor types.

In this study, the 3-year in-field control rate using IMRT was $52 \%$, which was comparable or superior to the approximately $50 \%$ reported in previous studies using 3Dconformal RT for LN oligometastases $(6,7)$. In contrast, a control rate of around $80 \%$ was reported for SRT $(2,8-12)$. SRT is usually preferred for local control because of its ability to provide a high dose focused on a small lesion. However, its use is typically limited to lesions $<3 \mathrm{~cm}$ in diameter because of the risk of adverse reactions (13). In addition, SRT cannot be applied to lesions near OARs such as the colon and intestine $(8,12,14,15)$. IMRT may therefore have an advantage over SRT for the treatment of oligo-recurrent lesions because of their larger size and location. 

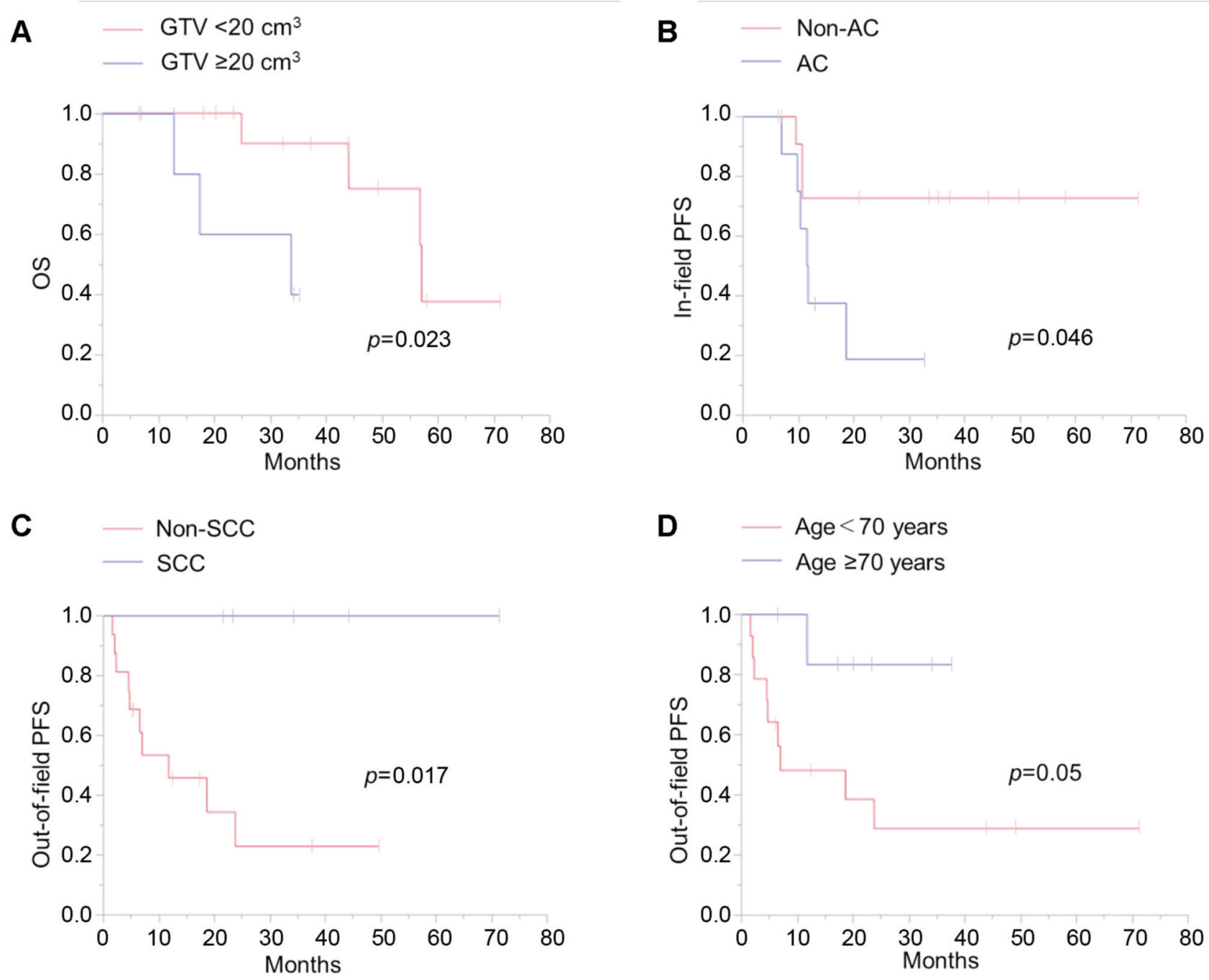

Figure 2. The effects of the potential prognostic factors on survival as analyzed by univariate analysis using log-rank test. A: Effect of gross tumor volume (GTV) on overall survival (OS). B: Effect of histopathological type, adenocarcinoma (AC) vs. non-AC, on in-field progression-free survival (PFS). C: Effect of histopathological type, squamous cell carcinoma (SCC) vs. non-SCC, on out-of-field PFS. D: Effect of age on out-of-field PFS.

The 3-year OS rate in the current study was $75 \%$. We believe this survival rate was relatively favorable. However, various cancer types were included in this study, making it difficult to compare the survival rate with previous studies. Although statistical analysis showed no significant difference between including and not including the regional $\mathrm{LN}$ area in a radiation field, among the 17 patients who received IMRT including the regional LN area, only one developed a newly arising tumor within the irradiated LN area. IMRT might therefore contribute to suppressing recurrence from the LN area, leading to a favorable OS rate. Irradiation to the regional LN area is one of the advantages of IMRT over SRT.

It is generally difficult to apply SRT to tumors $>3 \mathrm{~cm}$ or those near radiosensitive organs such as the intestine $(8,12$ -
14). In this study, the median GTV was $9.6 \mathrm{~cm}^{3}$ (range $=1.4$ $80 \mathrm{~cm}^{3}$ ) and eight patients had a GTV $>14 \mathrm{~cm}^{3}$ (equivalent to a sphere $3 \mathrm{~cm}$ in diameter). The tumors were controlled in five $(63 \%)$ of these eight patients with no acute or late adverse event, including in the two patients with tumors located close to the intestine. These results suggest that IMRT might be a potential treatment option in patients with tumors unsuitable for SRT because of their size and location.

In general, a high RT dose is associated with a better prognosis, and the dose to the GTV was significantly associated with better in-field PFS and OS rates in our study. A dose of $>60-70$ Gy is required to control solid tumors using conventional fraction RT (13), compared with a median dose to GTV in this study of $60 \mathrm{~Gy}$ (range $=50-70$ 
Gy). In-field PFS might have been improved further if the dose to the GTV had been higher (>60 Gy). High-precision IMRT and image-guided RT may allow such dose escalation.

GTV was significantly associated with OS in the current study and previous studies of SRT (2, 7, 9, 15-19), indicating that GTV is a significant factor affecting OS. It is therefore recommended to perform RT before tumor volume increases in order to prevent the tumor from acquiring the traits of expanding metastases/aggressiveness $(1,2)$.

Adenocarcinomas are generally considered to be less radiosensitive than other pathological types of tumors, such as squamous cell carcinoma. In line with this, adenocarcinoma was a poor prognostic factor for in-field PFS in the present study. In addition, RT failed in terms of in-field control in all four patients with adenocarcinoma treated with a dose to GTV of $\leq 60 \mathrm{~Gy}$. In contrast, treatment with the same doses did not achieve in-field control in only two out of eight patients with non-adenocarcinoma. These results suggest that a dose to the GTV of more than 60 Gy might be necessary in patients with oligo-recurrence of adenocarcinoma.

In this study, younger age was a poor prognostic factor in terms of out-of-field PFS. Other reports also identified younger age as a poor prognostic factor $(7,20)$. These results suggest that although we should consider differences in the backgrounds of the tumor types, older age itself may not be a reason to withhold IMRT. Indeed, RT may be the only treatment option for elderly patients because they are often not suitable for intensive chemotherapy and surgery.

Patients with squamous cell carcinoma had better out-offield PFS than those with non-squamous cell carcinoma. This might indicate that squamous cell carcinoma is less likely to be associated with out-of-field micrometastases at diagnosis of oligo-recurrence, and is more likely to be oligo-recurrent in the true sense.

This study had some limitations, including its retrospective nature, the small size of the cohort, and the heterogeneity of the primary pathogenesis. Given that OS and local control rates differ among different cancer and histopathological types, our results might have been affected by selection biases of the cancer type. Further studies are therefore needed to increase the sample size and to evaluate outcomes for each cancer type.

In conclusion, IMRT may represent a safe and effective treatment option in patients with LN oligo-recurrence, and may be a curative treatment in selected cases. Tumor dose escalation whilst sparing normal tissue using IMRT technology may improve OS and in-field PFS.

\section{Conflicts of Interest}

The Authors declare there were no conflicts of interest in regard to this study.

\section{Authors' Contributions}

Conceptualization: Akiko Sato and Motoko Omura. Data curation: Akiko Sato, Yumiko Minagawa, Yuki Mukai and Motoko Omura. Formal analysis: Akiko Sato, Motoko Omura and Toshihiro Misumi. Investigation: Akiko Sato, Motoko Omura, Yumiko Minagawa, Kengo Matsui, Ryosuke Shirata, Hideyuki Hongo, Harumitsu Hashimoto and Toshihiro Misumi. Methodology: Akiko Sato, Motoko Omura and Toshihiro Misumi. Project administration: Akiko Sato, Motoko Omura. Supervision: Motoko Omura, Tomio Inoue and Masaharu Hata. Writing - original draft: Akiko Sato. Writing - editing: Akiko Sato and Motoko Omura.

\section{Acknowledgements}

The Authors thank Dr. Yasuhito Sasaki, Director of Research Center for Radiation Oncology, Shonan Kamakura General Hospital Affiliated Clinical Research Center, for kind support and guidance based on his extensive knowledge.

\section{References}

1 Hellman S and Weichselbaum RR: Oligometastases. J Clin Oncol 13: 8-10, 1995. PMID: 7799047. DOI: 10.1200/JCO. 1995.13.1.8

2 Tree AC, Khoo VS, Eeles RA, Ahmed M, Dearnaley DP, Hawkins MA, Huddart RA, Nutting CM, Ostler PJ and van As NJ: Stereotactic body radiotherapy for oligometastases. Lancet Oncol 14: e28-37, 2013. PMID: 23276369. DOI: 10.1016/ S1470-2045(12)70510-7

3 Niibe $\mathrm{Y}$ and Hayakawa K: Oligometastases and oligorecurrence: the new era of cancer therapy. Jpn J Clin Oncol 40: 107-111, 2010. PMID: 20047860. DOI: 10.1093/jjco/hyp167.

4 Therasse P, Arbuck SG, Eisenhauer EA, Wanders J, Kaplan RS, Rubinstein L, Verweij J, Van Glabbeke M, Van Oosterom AT, Christian MC and Gwyther SG: New guidelines to evaluate the response to treatment in solid tumors. J Natl Cancer Inst 92: 205-216, 2000. PMID: 10655437. DOI: 10.1093/jnci/92.3.205

5 National Cancer Institute: Common Terminology Criteria for Adverse Events (CTCAE) Version 4.0. Available at: https://ctep.cancer.gov/protocolDevelopment/electronic_applicati ons/ctc.htm\#ctc_40 [Last accessed on May $14^{\text {th }}, 2020$ ]

6 Jingu K, Niibe Y, Yamashita H, Katsui K, Matsumoto T, Nishina T and Terahara A: Re-irradiation for oligo-recurrence from esophageal cancer with radiotherapy history: a multi-institutional study. Radiat Oncol 12: 146, 2017. DOI: 10.1186/s13014-017-0882-0

7 Seol KH, Lee JE, Cho JY, Lee DH, Seok Y and Kang MK: Salvage radiotherapy for regional lymph node oligo-recurrence after radical surgery of non-small cell lung cancer. Thorac Cancer 8: 620-629, 2017. PMID: 28906073. DOI: 10.1111/17597714.12497

8 Bignardi M, Navarria P, Mancosu P, Cozzi L, Fogliata A, Tozzi A, Castiglioni S, Carnaghi C, Tronconi MC, Santoro A and Scorsetti M: Clinical outcome of hypofractionated stereotactic radiotherapy for abdominal lymph node metastases. Int J Radiat Oncol Biol Phys 81: 831-838, 2011. PMID: 20800375. DOI: 10.1016/j.ijrobp.2010.05.032

9 Choi CW, Cho CK, Yoo SY, Kim MS, Yang KM, Yoo HJ, Seo YS, Kang JK, Lee DH, Lee KH, Lee ED, Rhu SY, Choi SC, 
Kim MH and Kim BJ: Image-guided stereotactic body radiation therapy in patients with isolated para-aortic lymph node metastases from uterine cervical and corpus cancer. Int J Radiat Oncol Biol Phys 74: 147-153, 2009. PMID: 18990511. DOI: 10.1016/j.ijrobp.2008.07.020

10 Wang H-H, Zaorsky NG, Meng M-B, Zeng X-L, Deng L, Song Y-C, Zhuang H-Q, Li F-T, Zhao L-J, Yuan Z-Y, Wang P and Hao $\mathrm{X}-\mathrm{S}$ : Stereotactic radiation therapy for oligometastases or oligorecurrence within mediastinal lymph nodes. Oncotarget 7: 18135-18145, 2016. PMID: 26919113. DOI: 10.18632/ oncotarget.7636

11 Kim M-S, Yoo SY, Cho CK, Yoo HJ, Yang KM, Kang JK, Lee DH, Lee JI, Bang HY, Kim MS and Kang HJ: Stereotactic body radiotherapy for isolated para-aortic lymph node recurrence after curative resection in gastric cancer. J Korean Med Sci 24: 488492, 2009. PMID: 19543514. DOI: 10.3346/jkms.2009.24.3.488

12 Kim M-S, Choi C, Yoo S, Cho C, Seo Y, Ji Y, Lee D, Hwang D, Moon S, Kim MS and Kang H: Stereotactic body radiation therapy in patients with pelvic recurrence from rectal carcinoma. Jpn J Clin Oncol 38: 695-700, 2008. PMID: 18723850. DOI: $10.1093 / \mathrm{jjco} / \mathrm{hyn} 083$

13 JASTRO Guideline 2016 for Radiotherapy Treatment Planning (Guidelines of Japanese Radiation Treatment Planning). Tokyo: Kanehara Co., Ltd., 2016. (In Japanese).

14 Seo YS, Kim M-S, Yoo H-J and Jang W-I: Stereotactic body radiotherapy for oligo-recurrence within the nodal area from colorectal cancer. World J Gastroenterol 20: 2005-2013, 2014. PMID: 24587675. DOI: 10.3748/wjg.v20.i8.2005

15 Conde-Moreno AJ, Lopez-Guerra JL, Macias VA, Vazquez de la Torre ML, Samper Ots P, San Jose-Maderuelo S, Pastor Peidro J, Lopez-Torrecilla J and Exposito-Hernandez J: Spanish Society of Radiation Oncology clinical guidelines for stereotactic body radiation therapy in lymph node oligometastases. Clin Transl Oncol 18: 342-351, 2016. PMID: 26329294. DOI: 10.1007/ s12094-015-1383-y
16 Bae SH, Kim M-S, Cho CK, Kang J-K, Kang HJ, Kim YH, Shin U-S, Moon SM and Lee DH: High dose stereotactic body radiotherapy using three fractions for colorectal oligometastases. J Surg Oncol 106: 138-143, 2012. PMID: 22297789. DOI: $10.1002 /$ jso. 23058

17 Navarria P, De Rose F and Ascolese AM: SBRT for lung oligometastases: Who is the perfect candidate? Reports Pract Oncol Radiother 20: 446-453, 2015. PMID: 26696785. DOI: 10.1016/j.rpor.2014.11.005

18 Milano MT, Katz AW, Zhang H and Okunieff P: Oligometastases treated with stereotactic body radiotherapy: Long-term followup of prospective study. Int J Radiat Oncol Biol Phys 83: 878886, 2012. PMID: 22172903. DOI: 10.1016/j.ijrobp.2011.08.036

19 Yamashita H, Jingu K, Niibe Y, Katsui K, Matsumoto T, Nishina $\mathrm{T}$ and Terahara A: Definitive salvage radiation therapy and chemoradiation therapy for lymph node oligo-recurrence of esophageal cancer: a Japanese multi-institutional study of 237 patients. Radiat Oncol 12: 38, 2017. PMID: 28219406. DOI: 10.1186/s13014-017-0780-5

20 Hong J-H, Tsai C-S, Lai C-H, Chang T-C, Wang C-C, Chou H$\mathrm{H}$, Lee SP and Hsueh S: Recurrent squamous cell carcinoma of cervix after definitive radiotherapy. Int J Radiat Oncol Biol Phys 60: 249-257, 2004. PMID: 15337563. DOI: 10.1016/j.jirobp. 2004.02.044

Received April 22, 2020

Revised May 12, 2020

Accepted May 15, 2020 